Revue d'histoire du XIXe siècle

Société d'histoire de la révolution de 1848 et des

révolutions du XIXe siècle

$23 \mid 2001$

Nouvelles approches en histoire économique

\title{
Annie STORA-LAMARRE [dir.], Archives de la peur. Les "populations à risque" dans la Franche-Comté au XIXe siècle
}

Presses universitaires franc-comtoises/Annales littéraires de l'université de Franche-Comté, 2000, 190 p.

Jean-Claude Caron

\section{(2) OpenEdition}

Journals

Édition électronique

URL : http://journals.openedition.org/rh19/327

DOI : 10.4000/rh19.327

ISSN : $1777-5329$

Éditeur

La Société de 1848

Édition imprimée

Date de publication : 1 décembre 2001

Pagination : 248-253

ISSN : 1265-1354

Référence électronique

Jean-Claude Caron, "Annie STORA-LAMARRE [dir.], Archives de la peur. Les "populations à risque" dans la Franche-Comté au XIXe siècle », Revue d'histoire du XIXe siècle [En ligne], 23 | 2001, mis en ligne le 15 octobre 2002, consulté le 22 septembre 2020. URL : http://journals.openedition.org/rh19/327 ; DOI : https://doi.org/10.4000/rh19.327

Ce document a été généré automatiquement le 22 septembre 2020.

Tous droits réservés 


\section{Annie STORA-LAMARRE [dir.], Archives de la peur. Les "populations à risque" dans la Franche-Comté au XIXe siècle}

Presses universitaires franc-comtoises/Annales littéraires de l'université de Franche-Comté, 2000, 190 p.

Jean-Claude Caron

Il convient tout d'abord de saluer une initiative trop rare prise par Annie StoraLamarre : offrir un espace éditorial à de jeunes chercheurs. Ce recueil est en effet constitué de sept contributions rédigées à partir de leur mémoire par des étudiants en maîtrise d'histoire contemporaine. À sa manière, ce collectif témoigne de la vigueur de la recherche universitaire et de la richesse des fonds d'archives départementaux. Les frontières qui déterminent le départ entre populations à risque et les autres ont fluctué entre le XIX ${ }^{e}$ et le XXe siècle. Il n'en reste pas moins que la notion de risque associée à des comportements ou à des pratiques relevant à la fois du regard social, moral ou judiciaire demeure une constante : à chaque époque ses risques et son traitement du risque. Sur ce point, les sources montrent leurs limites : elles produisent en effet une sur-représentation du traitement policier et judiciaire de ces populations "à risque", au détriment d'un discours émanant du voisinage, des solidarités informelles, d'une sociabilité parfois en rupture avec la sanction publique d'actes qui ne sont pas forcément réprouvés par les populations. Ce que ces sources nous donnent à voir et à comprendre touche de fait au "registre noir des malheurs du temps, qui s'accompagnent d'une promotion sans précédent de l'intériorisation de la conscience morale", à travers des récits qui se trouvent "au point d'intersection de subjectivités singulières et de l'organisation sociale" (A. Stora-Lamarre).

Tout commence par le décor : Besançon au XIX siècle est une ville qui, comme beaucoup d'autres, prend lentement conscience de la valeur de l'eau, de l'air, de la lumière, dans ce qui ne relève pas encore d'une politique de l'écologie, ni même de l'environnement, mais plus simplement d'un souci de vaincre et l'épidémie physique et 
l'épidémie morale qui peuvent infester et contaminer la ville. L'éclairage apporté par Angélique Barreiros permet de comprendre la spécificité bisontine (une eau omniprésente, au risque de l'humidité et de la crue) qui entraîne les édiles à se préoccuper dès le Second Empire, puis sous la Troisième République, des quais du Doubs avec l'aide d'un mécène, Adolphe Veil-Picard. Plus largement, la lutte contre l'insalubrité entraîne la destruction de nombreux îlots dans les vieux quartiers de la ville --qui sont aussi ceux où se concentrent estaminets et maisons closes.

L'aménagement de promenades a été plus précoce, dès la Monarchie de Juillet, et se poursuit au fur et à mesure de la récupération d'espaces dédiés à la défense de la ville, comme les glacis de Chamars. La lutte contre le miasme s'inscrit aussi dans le souci d'un air plus pur. La conquête de l'eau courante se traduit par de lourds travaux (aqueduc captant les eaux d'Arcier) et la multiplication des bornes-fontaines. Là encore, l'innovation se produit sous le Second Empire. Besançon n'est pas épargnée par les épidémies : choléra (1854), mais surtout typhoïde, véritablement récurrente.

L'inégalité sociale se révèle par le taux de mortalité de maladies comme la phtisie --qui fait des ravages dans la population ouvrière, notamment horlogère--, la tuberculose ou la syphilis. Si les causes sont hétérogènes, les conséquences sont homogènes. Liées aux effets de l'alcoolisme, ces maladies ou ces épidémies participent à l'élaboration d'une image du peuple souffrant, mais aussi du peuple coupable, générateur d'une peur sociale.

Aux marges morales de la ville, mais parfois en son cœur physique, figurent des individus qui sont plus tolérés que réellement acceptés. Ainsi des prostituées étudiées par Sylvine Jeantout, des naturalisés, présentés par Aline Voisin, ou des récidivistes, observés par Sébastien Bertin. Pas de ville sans ses prostituées : Besançon, ville de garnison et, à un moindre degré à cette époque, ville universitaire, concentre deux éléments importants de ce que Alain Corbin a qualifié de "prolétariat sexuel". La municipalité bisontine s'inscrit parfaitement dans le courant moralisateur qui parcourt la France du XIX ${ }^{e}$ siècle, en particulier celle de la III République : condamnation morale, réglementarisme croissant, mais tolérance des maisons du même nom, et même encouragement à regrouper ces filles perdues en des lieux plus faciles à surveiller et à contrôler, policièrement et sanitairement, avec la peur de la diffusion du mal vénérien, ce mal qui affaiblit durablement la "race". Le dispensaire de Bellevaux constitue la plaque tournante de ce contrôle sanitaire, d'autant qu'on y trouve aussi une prison qui accueille les prostituées condamnées. Même les isolées sont soumises au contrôle, la hantise restant les insoumises, filles clandestines que la police traque plus que les autres. Des centaines de contraventions sont dressées chaque année, bon indicateur de la présence de la prostitution dans la ville. Les bordels croissent dans la rue Sachot (une dizaine pour cette petite rue) et aux environs, du côté de Battant, prototype du quartier populaire. Besançon compte environ une centaine de prostituées au début des années 1870, dont $\mathrm{S}$. Jeantout décrit le quotidien avec une grande précision.

S'ils ne sont pas des réprouvés au même titre que les prostituées, les naturalisés font cependant l'objet d'un soupçon permanent. Ces individus sont en effet des étrangers dont la naturalisation n'a pas éteint d'un coup l'origine. Le fait que les deux tiers des étrangers présents à Besançon dans les années 1860-1880 soient originaires de Suisse (Genève, Neuchâtel, La Chaux-de-Fonds) ne joue pas nécessairement en faveur d'une assimilation plus aisée que déterminerait une proximité géographique, linguistique et culturelle. Les Suisses sont avant tout des rivaux, notamment dans l'horlogerie. Dans la 
partie nord du Doubs (Montbéliard), l'intégration des Alsaciens au lendemain de la défaite et de l'annexion ne se révèle pas plus facile. Reste le cas des Italiens, dont le flux devient important au tournant des deux siècles : eux aussi sont des rivaux, et pas seulement pour le travail, puisqu'il s'agit pendant longtemps d'une immigration essentiellement masculine. Malgré les facilités croissantes qu'offre la législation française (ainsi avec la loi de 1889 sur la nationalité), les demandes de naturalisation restent peu nombreuses. Le climat xénophobe des années 1890, la chasse au travail illégal, le rejet de ceux qui sont considérés comme des "jaunes" par certains syndicats, la propagande, y compris à gauche, en faveur du travail réservé aux nationaux, autant d'arguments qui ne militent pas en faveur d'une intégration rapide, pourtant souhaitée par les autorités pour lutter contre le déclin démographique du pays. De plus, une demande de naturalisation ne signifie pas son acceptation : le postulant doit satisfaire à la loi sur le recensement militaire et démontrer de réelles qualités morales --certificats à l'appui. Mieux vaut avoir aussi de bonnes mœurs matrimoniales et de bonnes mœurs politiques. Enfin, il va de soi que bénéficier de solides recommandations, ou justifier d'un talent spécial ou d'une activité industrielle enrichissante pour la nation, constituent autant de facteurs favorables à la naturalisation.

Les récidivistes, quant à eux, sont l'objet d'un discours particulier qui insiste sur la récurrence de la faute, et pousse à en identifier les causes, dans une dialectique qui oppose l'individu à la société. Si la misère, le désespoir, la déchéance sociale constituent un ordre d'explication qui se situe du côté des responsabilités de la société et de l'échec de son traitement de la délinquance --la prison, école du crime--, l'atavisme, la perversité, le vice font pencher la balance du côté de la responsabilité de l'individu, dans une lecture lombrosienne, avouée ou non, des causes de la criminalité --avec la notion de "criminel-né". Les récidivistes du canton de Pontarlier qu'étudie S. Bertin pour les années 1885-1900 sont plutôt moins nombreux que la moyenne nationale, une partie relevant de la lutte contre la contrebande, très active dans cette région frontalière. Mais le vagabondage et la mendicité sont également des causes majeures que l'on retrouve à l'échelle nationale. Pour autant, le débat fait rage autour du traitement judiciaire le mieux adapté à un comportement d'autant plus réprouvé qu'il est lié à une population masculine flottante, et donc inquiétante. Aussi la loi sur la relégation de 1885 est-elle promptement appliquée, mais assez rarement, alors que la loi sur la libération conditionnelle (1885) et celle sur le sursis (1891) permettent de disposer d'un arsenal où se côtoient la logique de la sanction et celle du rachat. Globalement, le taux de récidive enregistre une baisse tendancielle, quoique limitée. Il est vrai que, hier comme aujourd'hui, les chiffres sur l'évolution de la délinquance ou de la criminalité demeurent d'une interprétation délicate.

Le dépassement de la norme obéit à une échelle de gravité qui va de la simple contravention au crime le plus grave. La presse, qu'étudie Manuel Nardin, est le miroir le plus constant de cette peur sociale que suscite le crime. Le Petit comtois, principal quotidien bisontin sous la III ${ }^{e}$ République, consacre une place importante au fait divers en général, et au récit de crime en particulier. Il publie et commente des statistiques sur la délinquance et la criminalité, incrimine des lieux de débauche et des comportements "criminogènes" (l'alcoolisme, la pornographie), se fait le reflet de peurs nationales (ainsi des jeunes au temps des bandes d'apaches). Le crime hors norme bénéficie d'un traitement lui aussi hors norme : les mots de la violence, d'une crudité absolue, y côtoient les jugements moraux sur l'évolution de la société. La représentation du crime en passe par une mise en scène où affleure la fictionnarisation 
de l'événement. Tous les criminels, pourtant, ne relèvent pas du même traitement judiciaire : l'infanticide, objet des recherches d'Héléna Boffi, bénéficie d'une certaine forme de compréhension (du moins les jugements des cours d'assises en témoignentils), alors que "l'homo sanguinalis", au cœur du travail de Damiens Gerbier, constitue la figure du mal absolu. Les 224 cas d'infanticides relevés par H. Boffi pour le XIX ${ }^{e}$ siècle lui permettent de dresser le portrait de ces mères qui tuent leur enfant nouveau-né. Si les causes sont classiques (misère, isolement, pression sociale, abandon par le pèreséducteur), les mots pour le dire fournis par la source judiciaire donnent à entendre ces voix dont le discours, même tronqué, même normalisé, demeure une des seules voies d'accès à la parole populaire et, de plus, féminine. Qu'on l'appelle rupture de l'honneur ou poids de l'opinion, le sentiment individuel ou collectif qui entoure la divulgation d'une grossesse conçue en dehors du mariage constitue, surtout en milieu rural, une tache infamante. L'infanticide apparaît alors comme la réponse, apparemment disproportionnée, au déshonneur, à la perte de réputation qu'entraîne inévitablement une naissance illégitime. H. Boffi suit dans leur chronologie les événements de la courte séquence qui va de l'accouchement, secret, silencieux, solitaire, sans même parler de la douleur, à la mise à mort du nouveau-né. Elle montre les lieux, les méthodes. Cela explique aussi la relative clémence des discours qui s'ordonnent autour de l'acte. Si la condamnation morale comme judiciaire demeure la règle, elle connaît des exceptions et surtout des circonstances atténuantes. Au reste, un tiers des femmes jugées dans le Doubs sont acquittées ; au total, près de la moitié ne va pas en prison ou y reste deux ans au maximum. C'est autant la rupture des canons de la morale dominante qui est condamnable (une sexualité hors mariage, la débauche, à l'image de la fille facile) que le meurtre du nouveau-né en lui-même : du moins la place de l'enfant n'y est-elle pas centrale, pas plus que ne l'est, du reste, celle de l'homme, grand absent de ces procès. Le crime de sang qui accompagne la figure de "l'homo sanguinalis" incarne le sommet de la violence barbare. La trentaine de cas étudiés par D. Gerbier --3 femmes, 29 hommes-- pour le département du Doubs dans la deuxième moitié du XIX ${ }^{e}$ siècle permet de saisir à la fois le fonctionnement de la justice et l'élaboration d'une représentation du crime. Les dossiers d'archives autorisent la reconstitution de la chronologie d'actes criminels, telle que la justice l'a produite et à partir de laquelle elle a accompli sa mission. Soit la localisation du lieu du crime, le spectacle de la violence du crime-- décrite avec des mots qui ne sont pas sans rappeler ceux de la presse --et l'inventaire des outils du crime, la stratégie d'échappement du criminel et ses lieux de cachette ou d'exil. Puis viennent les circonstances de l'arrestation du criminel, la nature des aveux et l'élaboration des alibis, la confrontation de ce qui relève de son comportement public et de son comportement privé. Mots, postures, gestes, douleur ou colère, revendication ou négation de l'acte, la richesse des actes d'accusation et des procès-verbaux d'interrogatoires donne à comprendre la construction du discours judiciaire, qui, parfois, est dès l'instruction un discours vindicatoire, à tout le moins moralisateur, dans une logique de recherche d'adéquation avec le registre de la pénalité.

La richesse de ce recueil n'épuise pas la richesse du questionnement des sources utilisées. Il n'est pas exempt, parfois, de quelques maladresses ou d'insuffisances dans le questionnement de l'archive : il s'agit, en somme, de premiers pas dans la recherche qui incitent le lecteur plus expérimenté à se souvenir de ce qu'il a lui-même produit dans les mêmes circonstances. On mesure, à lire ces contributions, le formidable élargissement des objets d'histoire : il n'est nul besoin de citer des noms, ni même de 
retracer la généalogie de cet élargissement. Mieux vaut se souvenir que, dans un passé encore proche, l'histoire sociale des comportements "à risque" relevait encore de la non-historicité. On y voyait au mieux un aspect sociologique, voire une suite d'études de cas intéressant, par exemple, l'historien du droit ou l'anthropologue. Ce recueil témoigne que, grâce à une réelle mise à niveau bibliographique d'une part, et à un vrai travail méthodologique et épistémologique d'autre part, un débutant dans la recherche historique peut tirer profit de ces objets dont le traitement nécessite une authentique sensibilité historique. 